ordinarily large bathochromic shift of the rotatory dispersion extrema $(+39 \mathrm{~m} \mu$, which must be attributed to a very strong axial character ${ }^{11}$ of the acetoxy group, not anticipated by inspection of DREIDING models ${ }^{12}$. Even more surprising is the inversion of the sign of the Cotton effect of $V$, an observation for which we can offer no ready explanation other than to propose a substantial conformational deformation; rotatory contributions of freerotational rotomers of the acetoxy group may also need to be considered. The $16 \alpha$-acetoxy-17-ketone (VI) still possesses a positive Cotton effect and the bathochromic shift of its rotatory dispersion extremum (as compared to that of IV) suggests a slightly more axial orientation than in a $17 \beta$-acetoxy-16-ketone (II).

In conclusion, we can state that the optical rotatory dispersion data collected in the Table indicate the present difficulties in analyzing complex fused hydrindanones in terms of the octant rule and they also suggest some conformational distortion accompanying introduction of adjacent acetoxyl substituents ${ }^{13}$.

\title{
1,3-Dipolare Additionen der Thioketocarbene
}

Die erfolgreiche Verwendung von Ketocarbenen als 1,3-Dipole ${ }^{1}$ legte nahe, auch die Fähigkeit der geschwefelten Abkömmlinge zur 1,3-Dipolaren Addition ${ }^{2}$ zu prifen. Die von KIRMSE und HORNER ${ }^{3}$ beobachtete Bildung des Dithiafulven-Derivats II bei der Photolyse von I wies bereits auf eine 1,3-Addition des Phenyl-thioketocarbens an Phenyl-thioketen, das Produkt der WolffUmlagerung. Aus 1,2,3-Benzo-thiadiazol (III) erhielt JACOBSON ${ }^{4}$ beim Erhitzen auf $200-220^{\circ}$ das Thianthren (V), was auf eine Dimerisation des intermediären aromatischen Thioketocarbens IV deutet.

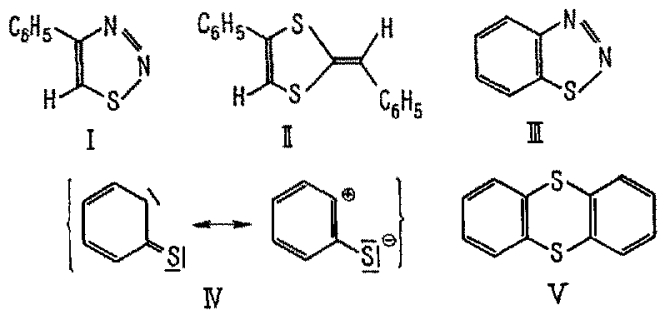

Unsere Versuche sprechen für eine erstaunliche Reaktionsträgheit von IV, verglichen mit dem Sauerstoffanalogen ${ }^{1}$. Beim Zerfall von III in Fumarsäurediäthylester oder $\alpha$-Naphtylisocyanat bei $220^{\circ}$ oder in siedendem Benzonitril liess sich die Bildung des Thianthrens nicht zugunsten einer Wechselwirkung von IV mit dem Lösungsmittel unterdrücken. Die für die Stickstoffabspaltung aus III nötige hohe Temperatur - wir fanden keine geeigneten Katalysatoren - begrenzt die Auswahl der Dipolarophilen. Einzig Verbindungen mit CS-Doppelbindung nehmen das Thioketocarben IV relativ glatt auf. Der Zerfall von III in Schwefelkohlenstoff im Autoklaven bei $220^{\circ}$ lieferte $84 \% 1,3$-Benzodithiol-2-thion (VI) ${ }^{5}$, das auf diesem Wege, ebenso wie das aus VI mit Lithiumaluminiumhydrid erhaltene Dithiobrenzcatechin, bequem zugänglich ist.

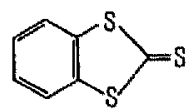

VI

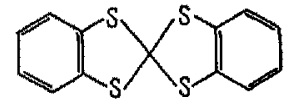

VI
Zusammentassung. Die optische Rotationsdispersion sowie die UV- und IR-Spektren der Epimeren von 17Acetoxy-16-keto- bzw. 16-Acetoxy-17-keto-14 $\beta$-steroiden wurden gemessen. Mögliche Beziehuingen zwischen Rotationsdispersion und Stereochemie der untersuchten Verbindungen werden diskutiert.

\section{DJerassi, J. Fishman, and T. Nambara}

Department of Chemistry. Stanford Uniwersity, Stanford (California), and Sloan Kettering Institute for Cancer Research, New York, August 23, 1967.

11 The work of J. FishmaN and T. Nambara, Chem. and Ind, 79 (1961), on 16-halogenated 14 $\beta$-17-ketosteroids supports the attribution of axial character to a $16 \beta$-substituent in this series. 12 A. Dreiding, Helv, chirn. Acta 42, 1339 (1959).

19 Acknowledgment. We are indebted to Mrs. RuTI REcords for the optical rotatory dispersion measurements and to the National Cancer Institute of the National Institutes of Health for financial support (grants No. CRTY-5061 and CY-3207).

Beim erneuten Zerfall von III in VI bei $200^{\circ}$ vermag die CS-Doppelbindung eine weitere Molekel des Thioketocarbens IV aufzunehmen. Das zu $69 \%$ Ausbeute entstandene Produkt ist vermutlich das noch nicht beschriebene Spirobi-[1,3-benzodithiol] (VII). Mit Phenylsenföl vereinigte sich zerfallendes III zu 38\% 2-Phenylimino1,3-benzodithiol (VIII), dessen Struktur durch unabhängige Synthese aus Dithiobrenzcatechin und Phenylisocyaniddichlorid in Gegenwart von Triäthylamin gesichert wurde.

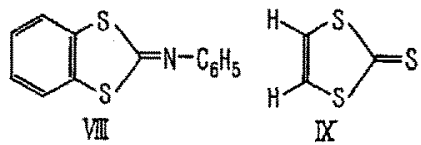

Auch bei der'Thermolyse oder Photolyse des 4-Phenyl1,2,3-thiadiazols (I) trat das Thioketocarben nicht mit Alkenen, Alkinen oder Nitrilen zusammen. Der Zerfall des unsubstituierten 1, 2, 3-Thiadiazols in Schwefelkohlenstoff bei $200^{\circ}$ lieferte $z u$ nur $0,2 \%$ das bekannte 1,3-Dithiol2-thion (IX) ${ }^{6}$

Summary. The aromatic thioketocarbene IV, generated by thermolysis of 1,2,3-benzothiadiazole at $200^{\circ}$, undergoes 1,3-dipolar additions with the CS-double bond of carbon disulfide, 1,3-benzodithiole-2-thion and phenyl isothiocyanate.

\section{R. HUISGEN und V. WeBERNDörfER}

Institut für Organische Chemie der Universität Minchen (Deutschland), 20. September 1961.

1 R. Hutsgen, H. Köng, G. Binsch und H. J. Sturm, Angew. Chem. $73,368(1061)$

2 Ubersicht: R. Hutsaen, Naturwiss. Rundschau 14, 43 (1961); Proc. Chem. Soc, $1961,357$.

3 W. Kirmse und L. Horner, Liebigs Ann. Chem. 614, 4 (1958).

4 P. Jacobson und H. Janssen, Liebigs Ann. Chem. 277, 209, 218 (1893).

* W. R. H. Hurtley und S. SMmLes, J. chem. Soc. [London] 1926, 1821, bereiteten VI aus Dithiobrenzcatechin, Schwefelkohlenstoff und Alkali.

6 F. Challenger, E. A. Mason, E. C. Holdsworth und R. Emmott, J. chem. Soc. [London] $1953,292$. 